Polymer Journal, Vol. 9, No. 5, pp 479-488 (1977)

\title{
Distribution Function of Polymers with and without Interactions. II. The Distribution Function of the Square Radius of Gyration of a Ring Chain
}

\author{
Takao Minato \\ Department of Pure and Applied Sciences, College of General Education, \\ University of Tokyo, Komaba, Meguro-ku, Tokyo 153, Japan.
}

(Received March 7, 1977)

\begin{abstract}
The characteristic function of the distribution function of $\boldsymbol{S}^{2}$ for a ring chain with interactions is obtained in closed form. The Fourier transformations of the characteristic function are performed numerically, in one-, two-, and threedimensional spaces and analytically in two-dimensional space. The expressions for the perturbed distribution functions valid for small $S^{2}$ values and large $S^{2}$ values are given. A simple variational technique is introduced to obtain the perturbed mean square radius of gyration in a closed form. It should be noted that, in two-dimensional spcce, there exists the negative critical value of $z$, below which no Fourier transformation of the characteristic function may be permitted. Here, $z$ is the usual excluded volume parameter.

KEY WORDS Interaction / Non-Gaussian / Path Integral / Radius of Gyration / Ring Chain /
\end{abstract}

In a previous paper (hereafter referred to as Part I), the present author ${ }^{1}$ proposed a method, based essentially on the first order perturbation theory, for obtaining the perturbed non-Gaussian distribution function such as that of the square distance of the center of mass from one fixed end of a chain. The purpose of this second article is to apply the same method for obtaining the perturbed distribution function of the square radius of gyration for a ring chain of infinite length, and make advanced commentation on the second moment of distribution and the phase change of a chain with an attractive interaction. This problem has attracted considerable interests in recent years. It has been supposed that a polymer with strong attractive interactions, will condense into a solid ball below a certain critical negative $z^{*}$ (the excluded volume parameter). Stockmayer ${ }^{2}$ has suggested that the critical value $z^{*}$ is $c a .-0.14$. Chikahisa ${ }^{3}$ suggests that the perturbation series for the expansion factor $\alpha^{2}$ for the mean square end-toend distance is divergent at $z^{*}$. Ptitsyn, et al., ${ }^{4}$ studied $z^{*}$ by the Monte Carlo method and obtained almost the same results as those by Stockmayer's. Oono ${ }^{5}$ showed that $z^{*}=0$, that is, a sufficiently long chain collapses just below $z=0$. This prediction is shown to be true by Saito $^{6}$ with the aid of an approximate theory. All of these comments are in reference to a three-dimensional polymer chain.

By using the Feynman path integral method, the present author has recently shown that, in two dimensional space, the mean square radius of gyration for a ring chain is proportional to $L^{-1 / 2}$ below negative $v_{\mathrm{c}}\left(\propto z^{*}\right)$ for purely attractive interactions of Gaussian type. ${ }^{7}$ Here, $v_{\mathrm{c}}$ corresponds to the amplitude of the delta-function, and $L$ is the total length of a chain.

Now, as shown in Part $I$, the distribution function $P\left(S^{2}\right)$ of the square radius of gyration for a ring chain is written as

$$
\begin{aligned}
P\left(\boldsymbol{S}^{2}\right)= & \int_{-\infty}^{+\infty} \frac{\mathrm{d} \eta}{2 \pi} \exp \left(-i \eta \boldsymbol{S}^{2}\right) \int_{r_{L}=0}^{r_{0}=0} \mathscr{C} \boldsymbol{r} \\
& \times \exp \left[-A \int_{0}^{L} \mathrm{~d} t \dot{\boldsymbol{r}}_{t}{ }^{2}+\frac{i \eta}{2 L^{2}} \int_{0}^{L} \int \mathrm{d} t \mathrm{~d} s\right. \\
& \left.\times\left(\boldsymbol{r}_{t}-\boldsymbol{r}_{s}\right)^{2}-B \int_{0}^{L} \int \mathrm{d} t \mathrm{~d} s V\left(\left|\boldsymbol{r}_{t}-\boldsymbol{r}_{\boldsymbol{s}}\right|\right)\right] \\
\equiv & \int_{-\infty}^{+\infty} \frac{\mathrm{d} \eta}{2 \pi} \exp \left(-i \eta \boldsymbol{S}^{2}\right) K(\eta ; L)
\end{aligned}
$$


with

$$
\begin{aligned}
K(\eta ; L)= & \int_{r_{0}=0}^{r_{L}=0} \mathscr{D} \exp \left[-A \int_{0}^{L} \mathrm{~d} t \dot{\boldsymbol{r}}_{t}^{2}\right. \\
& +\frac{i \eta}{2 L^{2}} \int_{0}^{L} \int \mathrm{d} t \mathrm{~d} s\left(\boldsymbol{r}_{t}-\boldsymbol{r}_{s}\right)^{2} \\
& \left.-B \int_{0}^{L} \int \mathrm{d} t \mathrm{~d} s V\left(\left|\boldsymbol{r}_{t}-\boldsymbol{r}_{s}\right|\right)\right]
\end{aligned}
$$

where $A=d / 2 l, B=1 / 2 l^{2}, \quad l$ is the length of a segment, $L$ is the total length of a chain, $d$ is the space dimensionality, and $V(r)$ is the interaction potential. When there exists no $V(\boldsymbol{r})$, the unperturbed characteristic function $K_{0}(\eta ; L)$ is given $\mathrm{by}^{8}$

$$
K_{0}(\eta ; L)=[(x / 2) / \sin (x / 2)]^{d}
$$

where $x^{2}=i \eta L / A$ and the subscript 0 refers to the unperturbed state. The Fourier transformation of eq 3 lead to the following asymptotic expressions: 8

$$
\begin{aligned}
\left\langle S^{2}\right\rangle_{0} P(t) \\
=\left(\frac{6}{\pi}\right)^{1 / 2} \sum_{n=0}\left[3(2 n+1)^{2} t^{-5 / 2}-t^{-3 / 2}\right] \\
\quad \times \exp \left[-3(2 n+1)^{2} / 2 t\right] \quad(d=1) \\
=36\left(\frac{3}{\pi}\right)^{1 / 2} \sum_{n=0}(n+1)^{2}\left[(n+1)^{2} t^{-7 / 2}-2^{-1} t^{-5 / 2}\right] \\
\quad \times \exp \left[-3(n+1)^{2} / t\right] \quad(d=2) \\
=12\left(\frac{2}{\pi}\right)^{1 / 2} \sum_{n=1}\left[1-2(2 n+1)^{2} t^{-1}\right. \\
\left.\quad+(2 n+1)^{4} 3^{-1} t^{-2}\right] \exp \left[-(2 n+1)^{2} / 2 t\right] \quad(d=3)
\end{aligned}
$$

for a small $t$ value, and

$$
\begin{aligned}
\left\langle S^{2}\right\rangle_{0} P(t)= & \frac{1}{3} \sum_{n=1}(-1)^{n+1} n^{2} \pi^{2} \\
& \times \exp \left(-n^{2} \pi^{2} t / 6\right) \quad(d=1) \\
= & \frac{2}{3} \sum_{n=1}\left(2 n^{4} \pi^{4} t / 3-3 n^{2} \pi^{2}\right) \\
& \times \exp \left(-n^{2} \pi^{2} t / 3\right) \quad(d=2) \\
= & \frac{1}{2} \sum_{n=1}(-1)^{n+1}\left(12 n^{2} \pi^{2}-9 n^{4} \pi^{4} t+n^{6} \pi^{6} t^{2}\right) \\
& \times \exp \left(-n^{2} \pi^{2} t / 2\right) \quad(d=3)
\end{aligned}
$$

for a large $t$ value (here, $t$ stands for the ratio of $\boldsymbol{S}^{2}$ to its statistical average, i.e., $\left.\left\langle\boldsymbol{S}^{2}\right\rangle_{0}=i L / 12\right)$.

In this work we have investigated the effect of the interactions on the above expressions. First, the closed expression for the perturbed characteristic function is evaluated in the same way as Part I is. Secondly, the Fourier transforms of characteristic functions are performed analytically for $d=2$ and numerically for $d=1$, 2 , and 3. Thirdly, in order to obtain the perturbed square radius of gyration $\left\langle S^{2}\right\rangle$ in a closed form, we have introduced a simple variational technique, and discussed the singular behavior of $P\left(\boldsymbol{S}^{2}\right)$ for negative $V(\boldsymbol{r})$ (attractive interactions).

\section{CALCULATIONS}

Derivations of the Perturbed Characteristic Function

As shown in Part I, the perturbed characteristic function $K(\eta ; L)$ is given by the following approximation:

$K(\eta ; L)=K_{0}(\eta ; L)$

$$
\begin{aligned}
& \times\left\langle\exp \left[-B \int_{0}^{L} \int \mathrm{d} t \mathrm{~d} s V\left(\left|\boldsymbol{r}_{t}-\boldsymbol{r}_{s}\right|\right)\right]\right\rangle \\
\simeq & K_{0}(\eta ; L) \\
& \times \exp \left[-B\left\langle\int_{0}^{L} \int \mathrm{d} t \mathrm{~d} s V\left(\left|\boldsymbol{r}_{t}-\boldsymbol{r}_{s}\right|\right)\right\rangle\right] \\
\equiv & K_{0}(\eta ; L) \exp (-U)
\end{aligned}
$$

where

$$
U=B\left\langle\int_{0}^{L} \int \mathrm{d} t \mathrm{~d} s V\left(\left|\boldsymbol{r}_{t}-\boldsymbol{r}_{s}\right|\right)\right\rangle
$$

which is the first order correction of $V(\boldsymbol{r})$ to $K_{0}(\eta ; L)$. Here the symbol $\langle\cdots\rangle$ denotes an average over $K_{0}(\eta ; L)$, i.e.,

$$
\begin{aligned}
\langle\cdots\rangle= & \int_{r_{0}=0}^{r_{L}=0} \mathscr{D} \boldsymbol{r} \exp \left[-A \int_{0}^{L} \mathrm{~d} t \dot{\boldsymbol{r}}_{t}^{2}\right. \\
& \left.+i \eta L^{\overline{2}} \int_{0}^{L} \int \mathrm{d} t \mathrm{~d} s\left(\boldsymbol{r}_{t}-\boldsymbol{r}_{s}\right)^{2}\right] \\
& / \int_{r_{0}=0}^{r_{L}=0} \mathscr{D} \boldsymbol{e x p}\left[-A \int_{0}^{L} \mathrm{~d} t \dot{\boldsymbol{r}}_{t}^{2}\right. \\
& \left.+\frac{i \eta}{2 L^{2}} \int_{0}^{L} \int \mathrm{d} t \mathrm{~d} s\left(\boldsymbol{r}_{t}-\boldsymbol{r}_{s}\right)^{2}\right]
\end{aligned}
$$

Let's introduce $Q$ defined as

$$
Q=\left\langle\exp -i \boldsymbol{k}\left(\boldsymbol{r}_{t}-\boldsymbol{r}_{s}\right)\right\rangle
$$

We can separate eq 8 into $d$-rectangular components. One of these components $Q_{\mathrm{x}}$ is easily calculated to give

$$
\begin{aligned}
Q_{\mathrm{x}} \simeq & \exp \left\{-(\beta / 4 \alpha)\left[2\left(1-\mathrm{e}^{-i \alpha|t-s|}\right)\right.\right. \\
& \left.\left.+\left(\mathrm{e}^{-i \alpha t}-\mathrm{e}^{-i \alpha s}\right)^{2}\right]{k_{\mathrm{x}}}^{2}\right\}
\end{aligned}
$$


Distribution Function of the Square Radius of Gyration

where $\alpha^{2}=-i \eta \mid A L, \beta=1 / 2 A$, and Real Part of $\alpha$ must be positive $(\operatorname{Re} \alpha>0)$. Thus we get from eq 47 in Part $\mathrm{I}$ for $U$ :

$$
U=\frac{B V L^{2}}{(2 \pi)^{d / 2}}(\alpha / \beta)^{d / 2}
$$

in the limit $L \rightarrow \infty$, where we used the delta function for $V(\boldsymbol{r})$, i.e., $V\left(\left|\boldsymbol{r}_{t}-\boldsymbol{r}_{s}\right|\right)=V \delta\left(\left|\boldsymbol{r}_{t}-\boldsymbol{r}_{s}\right|\right)$.

Now from the relations: $x^{2}=i \eta L / A$ and $\alpha^{2}=$ $-i \eta / A L$, the following equation is derived:

$$
\alpha^{2}=-x^{2} / L^{2}
$$

Taking the condition of $\operatorname{Re} \alpha>0$ into account, one obtains $\alpha=-i x / L$ for $\operatorname{Im} x>0$ and $\alpha=i x / L$ for $\operatorname{Im} x<0$, respectively. Then eq 10 is written in terms of $x$ as

$$
U=v( \pm i x)^{d / 2}
$$

where $v=V B L^{(4-d) 2} /(2 \pi \beta)^{d / 2}$, and the $\operatorname{sign}( \pm)$ means that $(-)$ corresponds to the case of $\operatorname{Im} x>0$ and $(+)$ to that of $\operatorname{Im} x<0$, respectively. From eqs 6 and 11 the perturbed characteristic function $K(\eta ; L)$ becomes

$$
K(\eta ; L)=[(x / 2) / \sin (x / 2)]^{d} \exp \left[-v( \pm i x)^{d / 2}\right]
$$

Table I. The numerical results from the integration of eq $14^{\prime}$ in one-dimension $(d=1)$

\begin{tabular}{lllll}
\hline \multicolumn{5}{c}{$\left\langle S^{2}\right\rangle_{0} P(t)$} \\
\hline$t$ & $v=0$ & $v=-0.05$ & $v=0.05$ & $v=0.1$ \\
\hline 0.1 & 0.0 & 0.0017 & 0.0 & 0.0 \\
0.2 & 0.1209 & 0.3915 & 0.0312 & 0.0066 \\
0.3 & 0.5100 & 1.0450 & 0.2194 & 0.0839 \\
0.4 & 0.8342 & 1.3256 & 0.4721 & 0.2419 \\
0.5 & 0.9737 & 1.3309 & 0.6486 & 0.3969 \\
0.6 & 0.9754 & 1.1950 & 0.7290 & 0.5037 \\
0.7 & 0.9101 & 1.0292 & 0.7388 & 0.5574 \\
0.8 & 0.8137 & 0.8607 & 0.7065 & 0.5710 \\
0.9 & 0.7137 & 0.7137 & 0.6535 & 0.5574 \\
1.0 & 0.6162 & 0.4800 & 0.5916 & 0.5290 \\
1.2 & 0.4517 & 0.3922 & 0.4690 & 0.4517 \\
1.4 & 0.3273 & 0.2599 & 0.3637 & 0.3721 \\
1.6 & 0.2363 & 0.1703 & 0.2798 & 0.3016 \\
1.8 & 0.1703 & 0.1093 & 0.2150 & 0.2430 \\
2.0 & 0.1227 & 0.0681 & 0.1655 & 0.1956 \\
2.4 & 0.0636 & 0.0214 & 0.0995 & 0.1281 \\
2.8 & 0.0328 & 0.0007 & 0.0617 & 0.0862 \\
3.4 & 0.0121 & 0.0 & 0.0325 & 0.0509 \\
4.0 & 0.0044 & & 0.0191 & 0.0329 \\
\hline
\end{tabular}

Table II. The numerical results from the integration of eq $14^{\prime}$ in two-dimensions $(d=2)$

\begin{tabular}{lllll}
\hline \multicolumn{5}{c}{$\left\langle S^{2}\right\rangle_{0} P(t)$} \\
\hline$t$ & $v=0$ & $v=-0.05$ & $v=0.05$ & $v=0.1$ \\
\hline 0.1 & 0.0 & 0.0 & 0.0 & 0.0 \\
0.2 & 0.0026 & 0.0098 & 0.0006 & 0.0001 \\
0.3 & 0.0917 & 0.2045 & 0.0387 & 0.0154 \\
0.4 & 0.3844 & 0.6663 & 0.2111 & 0.1105 \\
0.5 & 0.7396 & 1.0975 & 0.4774 & 0.2954 \\
0.6 & 0.9914 & 1.3202 & 0.7148 & 0.4963 \\
0.7 & 1.0964 & 1.3449 & 0.8590 & 0.6488 \\
0.8 & 1.0840 & 1.2435 & 0.9073 & 0.7323 \\
0.9 & 0.9990 & 1.0815 & 0.8839 & 0.7535 \\
1.0 & 0.8784 & 0.9021 & 0.8163 & 0.7294 \\
1.2 & 0.6214 & 0.5765 & 0.6302 & 0.6102 \\
1.4 & 0.4084 & 0.3392 & 0.4503 & 0.4679 \\
1.6 & 0.2563 & 0.1854 & 0.3087 & 0.4022 \\
1.8 & 0.1594 & 0.1328 & 0.2075 & 0.2466 \\
2.0 & 0.0927 & 0.0393 & 0.1389 & 0.1767 \\
2.4 & 0.0313 & & 0.0644 & 0.0941 \\
2.8 & 0.0101 & & 0.0333 & 0.0551 \\
3.4 & & & 0.0162 & 0.0303 \\
4.0 & & & 0.0102 & 0.0200 \\
\hline
\end{tabular}

Table III. The numerical results from the integration of eq $14^{\prime}$ in three-dimensions $(d=3)$

\begin{tabular}{lllll}
\hline \multicolumn{5}{c}{$\left\langle S^{2}\right\rangle_{0} P(t)$} \\
\hline$t$ & $v=0$ & $v=-0.05$ & $v=0.05$ & $v=0.1$ \\
\hline 0.1 & 0.0 & 0.0 & 0.0 & 0.0 \\
0.2 & 0.0 & 0.0002 & 0.0 & 0.0 \\
0.3 & 0.0143 & 0.0328 & 0.0059 & 0.0024 \\
0.4 & 0.1535 & 0.2735 & 0.0834 & 0.0439 \\
0.5 & 0.4878 & 0.7435 & 0.3112 & 0.1931 \\
0.6 & 0.8733 & 1.1939 & 0.6222 & 0.4321 \\
0.7 & 1.1462 & 1.4424 & 0.8871 & 0.6700 \\
0.8 & 1.2498 & 1.4699 & 1.0342 & 0.8346 \\
0.9 & 1.2100 & 1.3412 & 1.0600 & 0.9044 \\
1.0 & 1.0804 & 1.1333 & 0.9961 & 0.8924 \\
1.2 & 0.7345 & 0.6902 & 0.7450 & 0.7278 \\
1.4 & 0.4359 & 0.3594 & 0.4877 & 0.5167 \\
1.6 & 0.2373 & 0.1619 & 0.2973 & 0.3422 \\
1.8 & 0.1215 & 0.0588 & 0.1759 & 0.2210 \\
2.0 & 0.0596 & 0.0106 & 0.1044 & 0.1438 \\
2.4 & 0.0131 & & 0.0413 & 0.0679 \\
2.8 & 0.0026 & & 0.0212 & 0.0392 \\
3.4 & & & 0.0116 & 0.0228 \\
4.0 & & & 0.0079 & 0.0158 \\
\hline & & & &
\end{tabular}


Fourier Transformation of the Characteristic Function: Numerical Results

From eq 1 and 13 the perturbed distribution function $P\left(\boldsymbol{S}^{2}\right)$ is given as

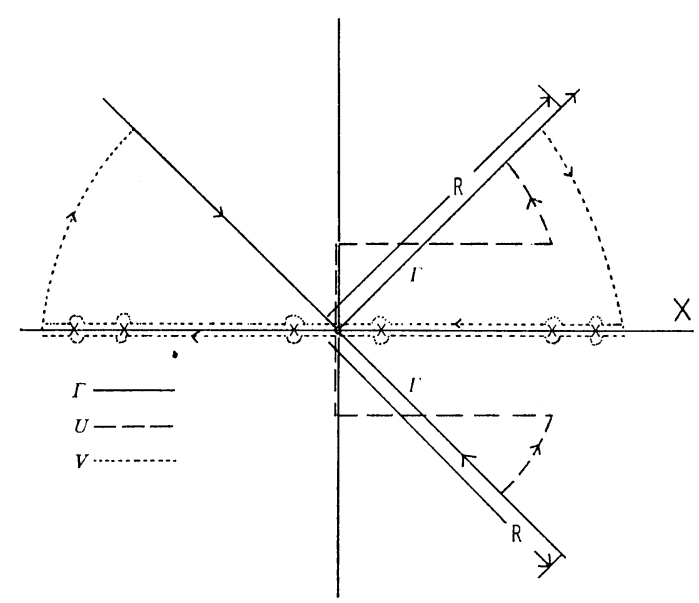

Figure 1. The integration paths chosen for the derivation of the asymptotic expressions for $P(t)$. The original path $\Gamma$ is deformed to become the path $U$, represented by the broken line for the derivation of eq 15, and to become the path $V$, represented by the dotted line for the derivation of eq 16.

$$
\begin{aligned}
P\left(\boldsymbol{S}^{2}\right)= & \int_{-\infty}^{+\infty} \frac{\mathrm{d} \eta}{2 \pi}[(x / 2) / \sin (x / 2)]^{d} \\
& \times \exp \left[-\mathrm{d} t x^{2} / 24-v( \pm i x)^{d / 2}\right] \\
\equiv & \underset{6 i \pi\left\langle S^{2}\right\rangle_{0}}{d} \int_{\Gamma(\langle)} \mathrm{d} x \frac{d^{d+1}}{\sin ^{d} x} \\
& \times \exp \left[-\mathrm{d} t x^{2} / 6-v( \pm 2 i x)^{d / 2}\right]
\end{aligned}
$$

where $\Gamma(\zeta)$ is the path of integration as shown in Figure $1^{1,12}$ and $t=S^{2} /\left\langle S^{2}\right\rangle_{0}=12 S^{2} / l L$. Equation $14^{\prime}$ was numerically calculated for certain values of $v$ as a function of $t$. Detailed numerical results are tabulated in Table I, II, and III for $d=1,2$, and 3 , respectively, and these are illustrated in Figure $1-3$. These figures show that the most probable peak of the distribution is pushed out and pulled in by repulsive forces $(v>0)$ and attractive forces $(v<0)$, respectively.

Fourier Transformation of the Characteristic

Function: Analytical Results

The complex integral in eq $14^{\prime}$ can be analytically performed only in two-dimensional space $(d=2)$. That is, this integral is evaluated by the deformation of the original path $\Gamma$ to appropriate paths as shown in Figure 1. Details can be seen in Part $\mathrm{I}$. For a small $t$ value, we obtain

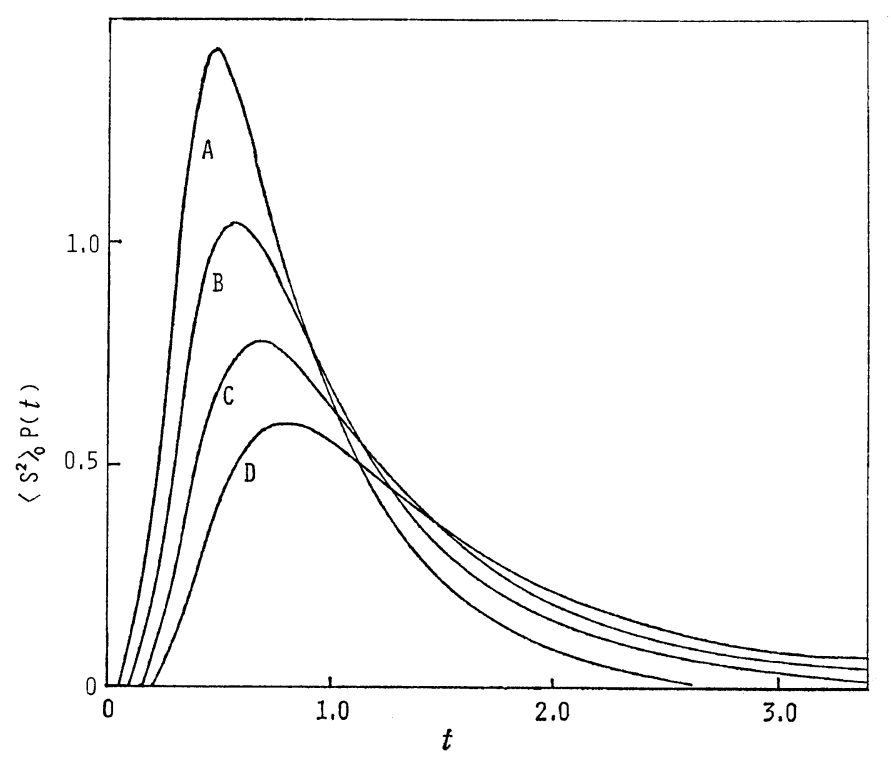

Figure 2. The one-dimensional distribution functions $\left\langle S^{2}\right\rangle_{0} P(t)$ as a function of $t$ for various values of $v$ : curve $\mathrm{A}, v=-0.05$; curve $\mathrm{B}, v=0.0$; curve $\mathrm{C}, v=0.05$; curve $\mathrm{D}, v=0.1$. 
Distribution Function of the Square Radius of Gyration

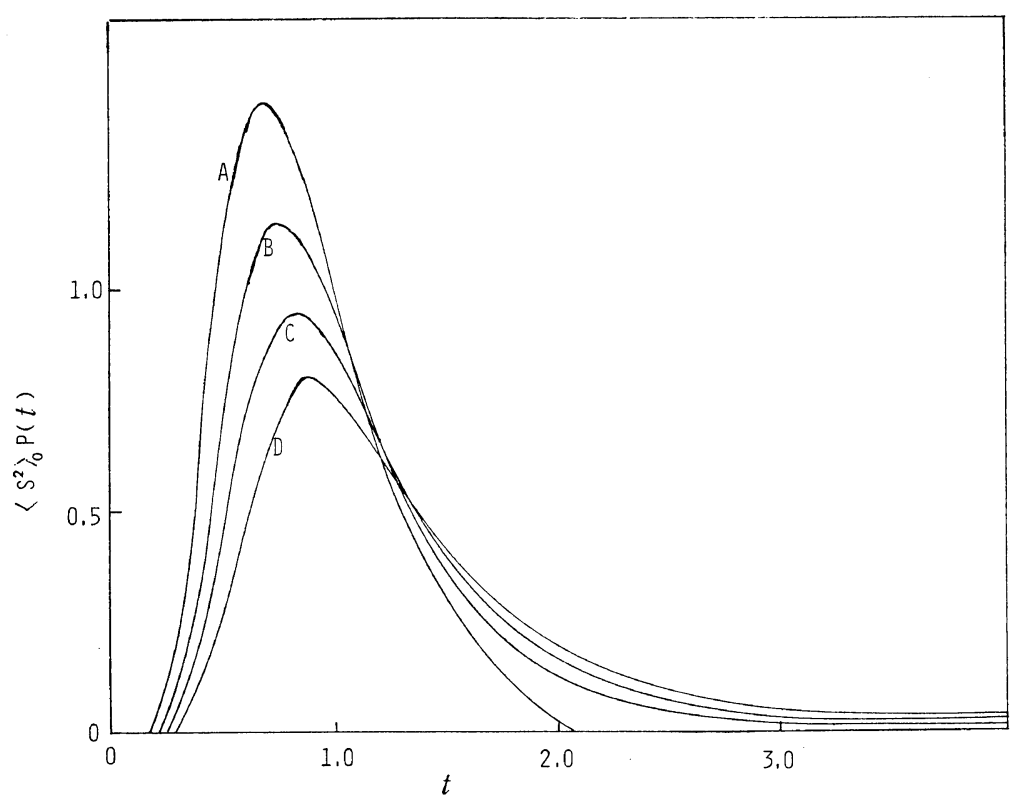

Figure 3. The two-dimensional distribution functions $\left\langle S^{2}\right\rangle_{0} P(t)$ as a function of $t$ for various values of $v$ : curve $\mathbf{A}, v=-0.05$; curve $\mathbf{B}, v=0.0$; curve $\mathrm{C}, v=0.05$; curve $\mathbf{D}, v=0.1$.

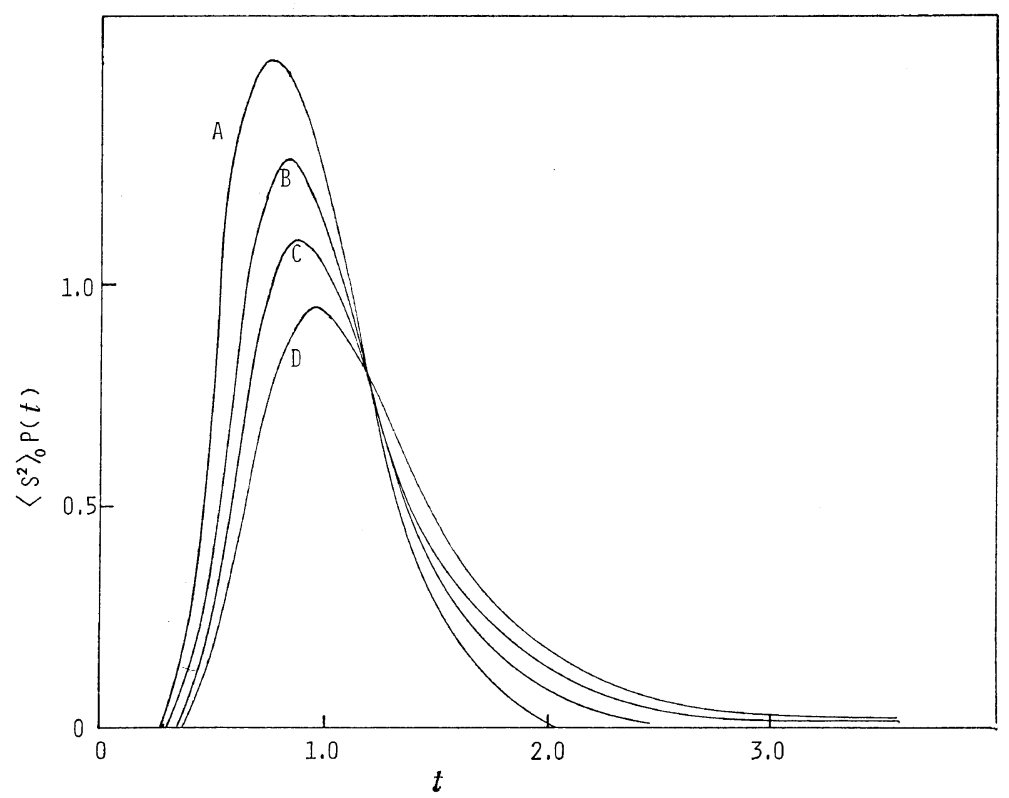

Figure 4. The three-dimensional distribution functions $\left\langle S^{2}\right\rangle_{0} P(t)$ as a function of $t$ for various values of $v$ : curve $\mathrm{A}, v=-0.05$; curve $\mathrm{B}, v=0.0$; curve $\mathrm{C}, v=0.05$; curve $\mathrm{D}, v=0.1$. 


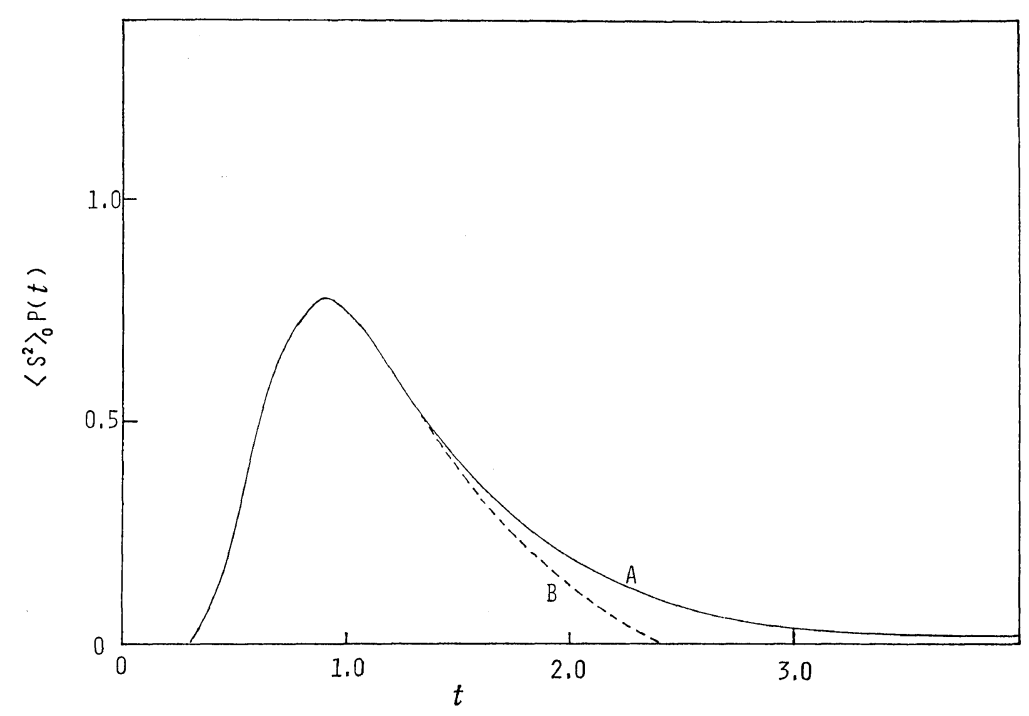

Figure 5. The comparison of the exact form with asymptotic form given by the leading term of eq 15 for $v=0.1$. Curve A: exact, curve B: eq 15 .

$$
\begin{array}{rl}
\left\langle\boldsymbol{S}^{2}\right\rangle_{0} & P\left(\boldsymbol{S}^{2}\right) \\
= & 36\left(\frac{3}{\pi}\right)^{1 / 2} \sum_{n=0}(n+1) \\
& \times\left[(n+1+v)^{3} t^{-7 / 2}-2^{-1}(n+1+v) t^{-5 / 2}\right] \\
& \times \exp \left[-3(n+1+v)^{2} / t\right]
\end{array}
$$

For a large $t$ value we get

$$
\begin{aligned}
\left\langle S^{2}\right\rangle_{0} P(t)= & \frac{2}{3} \sum_{n=1}\left[\left(2 n^{4} \pi^{4} t / 3-3 n^{2} \pi^{2}\right) \cos (2 n \pi v)\right. \\
& \left.+2 v \pi^{3} n^{3} \sin (2 n \pi v)\right] \exp \left(-n^{2} \pi^{2} t / 3\right) \\
& +\frac{v}{3} \pi^{-1 / 2}\left(\frac{3}{t}\right)\left[1+\frac{1}{2}\left(\frac{3}{t}\right)+\cdots\right] \\
& \times \exp \left(-3 v^{2} / t\right)
\end{aligned}
$$

It can be shown that the values of $\left\langle S^{2}\right\rangle_{0} P\left(S^{2}\right)$ as a function of $t$ numerically oalculated from eq $14^{\prime}$ are in close agreement with those calculated from eq 15 and 16 in the regions where eq 15 and 16 are respectively applicable. In particular the leading term in eq 15 , which is valid for a small $t$ value, describes fairly well the behavior of $P\left(S^{2}\right)$ when $t$ is relatively large. This situation is shown in Figure 5.

\section{DISCUSSIONS}

The Second Moment of the Distribution

Hereafter we shall consider only a two-dimen- sional chain. With the aid of eq 15 which is valid for small values of $t$, the perturbed second moment $\left\langle S^{2}\right\rangle$ is given by (see Appendix)

$$
\begin{aligned}
\langle t\rangle & =\left\langle\boldsymbol{S}^{2} /\left\langle\boldsymbol{S}^{2}\right\rangle_{0}\right\rangle=\left\langle 12 \boldsymbol{S}^{2} / l L\right\rangle \\
& =\int_{0}^{\gamma} \mathrm{d} t t P(t) / \int_{0}^{\gamma} \mathrm{d} t P(t) \propto(1+v)^{2} ; \quad \gamma=2(1+v)^{2}
\end{aligned}
$$

where we have taken the term $n=0$ only in eq 15 , and the integral region is cut off at $\gamma$ because $P(t)$ has negative values if $t>\gamma$. Thus $\left\langle S^{2}\right\rangle$ is written in the form

$$
\alpha_{\mathrm{s}}{ }^{2}=\frac{\left\langle\boldsymbol{S}^{2}\right\rangle}{\left\langle\boldsymbol{S}^{2}\right\rangle_{0}}=1+2 v+v^{2}
$$

This shows that the second moment, or, the expansion factor $\alpha_{\mathrm{s}}^{2}$, is expressed as a power series in the parameter $v$ up to the second order. From the definition of $v=V L A / 2 \pi l^{2}, v$ is expressed by the excluded volume parameter $z$ as $v=V L A / 2 \pi l^{2}=V L / 2 \pi l^{3}=z / 2 ; \quad z=V L / \pi l^{3} \quad(d=2)$

Therefore eq 18 becomes

$$
\alpha_{\mathrm{s}}^{2}=1+z+z^{2} / 4
$$

On the other hand, the first order perturbation theory of $\left\langle\boldsymbol{S}^{2}\right\rangle$ for a two-dimensional linear 
chain leads to ${ }^{9}$

$$
\alpha_{\mathrm{s}}^{2}=1+11 / 24 z-\cdots
$$

The coefficient of $z$ in eq 20 is about twice as large as that in eq 21 . Generally speaking, the coefficient of $z$ for a ring chain is larger than that for a linear chain as is so of a threedimensional chain, and this tendency may become more pronounced with a decrease in the space dimension. Since the exact value of the coefficient of $z$ for a two-dimensional ring chain has not been reported, we can make no comparison with eq 20.

Certain devices are necessary in order to obtain $\left\langle\boldsymbol{S}^{2}\right\rangle$ in a closed, nonperturbative form. Here, if we take

$$
\begin{aligned}
K_{\mathrm{T}}\left(C: \gamma_{j} ; L\right)= & \int_{r_{0}=0}^{r_{L}=0} \mathscr{D} \boldsymbol{r} \exp \left[-A \int_{0}^{L} \mathrm{~d} t_{\boldsymbol{r}_{t}}{ }^{2}\right. \\
& \left.+\frac{i C \eta}{2 L^{2}} \int_{0}^{L} \int \mathrm{d} t \mathrm{~d} s\left(\boldsymbol{r}_{t}-\boldsymbol{r}_{s}\right)^{2}\right]
\end{aligned}
$$

as the trial path integral, we may rewrite eq 2 in the form,

$$
\begin{aligned}
K(\eta ; L)= & K_{\mathrm{T}}(C: \eta ; L) \\
& \times\left\langle\operatorname { e x p } \left[(1-C) \frac{i \eta}{2 L^{2}} \int_{0}^{L} \int \mathrm{d} t \mathrm{~d} s\left(\boldsymbol{r}_{t}-\boldsymbol{r}_{s}\right)^{2}\right.\right. \\
& \left.\left.-B \int_{0}^{L} \int \mathrm{d} t \mathrm{~d} s V\left(\left|\boldsymbol{r}_{t}-\boldsymbol{r}_{s}\right|\right)\right]\right\rangle_{\mathrm{T}}
\end{aligned}
$$

where $C$ is the parameter to be chosen to minimize the free energy of a chain and the average $\langle\cdots\rangle_{\mathrm{T}}$ has the same meaning as that in eq 7 . Furthermore, we approximate eq 23 as

$$
K(\eta ; L) \cong K_{\mathrm{T}}(C: \eta ; L) \exp (M+N)
$$

where the integrals $M$ and $N$ are given as

$$
\begin{gathered}
\boldsymbol{M}=(1-C)\left\langle\frac{\text { in }}{2 L^{2}} \int_{0}^{L} \int \mathrm{d} t \mathrm{~d} s\left(\boldsymbol{r}_{t}-\boldsymbol{r}_{s}\right)^{2}\right\rangle_{\mathrm{T}} \\
N=-\left\langle B \int_{0}^{L} \int \mathrm{d} t \mathrm{~d} s V\left(\left|\boldsymbol{r}_{t}-\boldsymbol{r}_{\boldsymbol{s}}\right|\right)\right\rangle_{\mathrm{T}}
\end{gathered}
$$

To find $M$ we need $\left\langle\left(\boldsymbol{r}_{t}-\boldsymbol{r}_{s}\right)^{2}\right\rangle_{\mathrm{T}}$. This can be obtained by expanding the r.h.s. of eq 8 and eq 9 with respect to $\boldsymbol{k}$ and $\boldsymbol{k}_{\mathrm{x}}$ up to the order $\boldsymbol{k}^{2}$ and $\boldsymbol{k}_{\mathrm{x}}{ }^{2}$, respectively. Then using the relation $\left\langle 2\left(\boldsymbol{r}_{\mathrm{x} t}-\boldsymbol{r}_{\mathrm{x} s}\right)^{2}\right\rangle_{\mathrm{T}}=\left\langle\left(\boldsymbol{r}_{t}-\boldsymbol{r}_{s}\right)^{2}\right\rangle_{\mathrm{T}}$, we get $\left\langle\left(\boldsymbol{r}_{t}-\boldsymbol{r}_{s}\right)^{2}\right\rangle_{\mathrm{T}}=(\beta / \alpha)\left[2\left(1-\mathrm{e}^{-i \alpha|t-s|}\right)+\left(\mathrm{e}^{-i \alpha t}-\mathrm{e}^{-i \alpha s}\right)^{2}\right]$ in two-dimensional space where $\alpha^{2}=-i C \eta / A L$. The integral in $M$ is now easily performed and the expression simplifies to

$$
M=(1-C) C^{-1 / 2}(\beta A)( \pm i x)
$$

where $( \pm)$ has the same meaning as mentioned before. The integral in $N$ is obtained by the replacement of $x$ by $C^{1 / 2} x$ in eq 12:

$$
N=-v C^{1 / 2}( \pm i x)
$$

From eq $23-29 K(\eta ; L)$ becomes

$$
\begin{aligned}
K(\eta ; L)= & {\left[\left(C^{1 / 2} x / 2\right) / \sin \left(C^{1 / 2} x / 2\right)\right]^{2} } \\
& \times \exp \left\{-( \pm i x)\left[-w(C-1) C^{-1 / 2}+v C^{1 / 2}\right]\right\}
\end{aligned}
$$

where $w=\beta A$. Then $P\left(\boldsymbol{S}^{2}\right)$ is expressed as

$$
\begin{aligned}
P\left(S^{2}\right)= & \int_{-\infty}^{+\infty} \frac{\mathrm{d} \eta}{2 \pi}\left[\left(C^{1 / 2} x / 2\right) / \sin \left(C^{1 / 2} x / 2\right)\right]^{2} \\
& \times \exp \left\{-t x^{2} / 12\right. \\
& \left.-( \pm i x)\left[v C^{1 / 2}-w(C-1) C^{-1 / 2}\right]\right\} \\
\equiv & \frac{C^{-2}}{3 i \pi\left\langle S^{2}\right\rangle_{0}} \int_{\Gamma} \mathrm{d} x \frac{x^{3}}{\sin ^{2} x} \\
& \times \exp \left\{-t x^{2} / 3 C-2( \pm i x)\left[w\left(C^{-1}-1\right)+v\right]\right\}
\end{aligned}
$$

Since the distribution function which covers the whole range of $t$ cannot be obtained, we will derive the expression for $P\left(S^{2}\right)$ that is valid for small values of $t$. By replacing $t$ and $v$ with $t / C$ and $v-w\left(1-C^{-1}\right)$ in eq 15 , respectively, one finds immediately that

$$
\begin{aligned}
\left\langle S^{2}\right\rangle_{0} P(t) \propto & \left\{\left[1+v-w\left(1-C^{-1}\right)\right]^{3}(t / C)^{-7 / 2}\right. \\
& \left.-2^{-1}\left[1+v-w\left(1-C^{-1}\right)\right](t / C)^{-5 / 2}\right\} \\
& \times \exp \left\{-3 C\left[1+v-w\left(1-C^{-1}\right)\right]^{2} / t\right\}
\end{aligned}
$$

where the term $n=0$ is taken only in eq 15 .

Here, we notice that eq 31 can be regarded as the expression for the free energy of a ring chain with $S^{2}$ fixed. Then, neglecting $\ln$ terms, we get

$$
F \propto 3 C\left(u+w C^{-1}\right)^{2}
$$

with $u=1+v-w$ for the free energy $F$. Differentiating eq 32 with respect to $C, C=w / u$ is obtained. This gives

$$
\left\langle S^{2}\right\rangle_{0} P(t) \propto \exp (-12 u w / t)
$$

so that we have

$$
\left\langle\boldsymbol{S}^{2}\right\rangle \propto L(1+v-w) \propto L^{2}
$$




\section{T. Minato}

in the limit of $L \rightarrow \infty$ where $v \propto L$ was used. J. de Cloizeaux has shown that the mean square end-to-end distance $\left\langle R^{2}\right\rangle$ is proportional to $L^{2}$ for $d=2 .^{10}$ The result of eq 34 coincides with this, but does not agree with the numerical calculations of a lattice chain. ${ }^{11}$

\section{Singular Behavior of the Distribution Function}

We now consider the singular behavior of the distribution function represented by eq $14^{\prime}$ for negative values of $v$ (attractive forces). Before proceeding, a simple physical meaning is given for our approximation in eq $6 a-6 b$. The exponential part in the r.h.s. of eq $6 a$ may be expanded in powers of

$$
\int_{0}^{L} \int \mathrm{d} t \mathrm{~d} s V\left(\left|\boldsymbol{r}_{t}-\boldsymbol{r}_{\boldsymbol{s}}\right|\right)
$$

and this may be summed up again as follows:

$$
\begin{aligned}
\left\langle\exp \int_{0}^{L} \int V_{\mathrm{ts}} \mathrm{d} t \mathrm{~d} s\right\rangle=1 & +\left\langle\int_{0}^{L} \int \mathrm{d} s \mathrm{~d} t V_{\mathrm{ts}}\right\rangle+\frac{1}{2 !}\left\langle\int_{0}^{L} \cdots \int \mathrm{d} t \mathrm{~d} s \mathrm{~d} t^{\prime} \mathrm{d} s^{\prime} V_{\mathrm{ts}} V_{\mathrm{t}^{\prime} \mathrm{s}^{\prime}}\right\rangle \\
& \times \frac{1}{3 !}\left\langle\int_{0}^{L} \cdots \int \mathrm{d} t \mathrm{~d} s \mathrm{~d} t^{\prime} \mathrm{d} s^{\prime} \mathrm{d} t^{\prime \prime} \mathrm{d} s^{\prime \prime} V_{\mathrm{ts}} V_{\mathrm{t}^{\prime} \mathrm{s}^{\prime}} V_{\mathrm{t}^{\prime \prime} \mathrm{s}^{\prime \prime}}\right\rangle+\cdots \\
\simeq 1 & +\left\langle\int_{0}^{L} \int \mathrm{d} t \mathrm{~d} s V_{\mathrm{ts}}\right\rangle+\frac{1}{2 !}\left\langle\int_{0}^{L} \int \mathrm{d} t \mathrm{~d} s V_{\mathrm{ts}}\right\rangle\left\langle\int_{0}^{L} \int \mathrm{d} t^{\prime} \mathrm{d} s^{\prime} V_{\mathrm{t}^{\prime} \mathrm{s}^{\prime}}\right\rangle \\
& +\frac{1}{3 !}\left\langle\int_{0}^{L} \int \mathrm{d} t \mathrm{~d} s V_{\mathrm{ts}}\right\rangle\left\langle\int_{0}^{L} \int \mathrm{d} t^{\prime} \mathrm{d} s^{\prime} V_{\mathrm{t}^{\prime} \mathrm{s}^{\prime}}\right\rangle\left\langle{ }_{0}^{L} \int \mathrm{d} t^{\prime \prime} \mathrm{d} s^{\prime \prime} V_{\mathrm{t}^{\prime \prime} \mathrm{s}^{\prime \prime}}\right\rangle+\cdots \\
= & \exp \left\langle\int_{0}^{L} \int \mathrm{d} t \mathrm{~d} s V_{\mathrm{ts}}\right\rangle
\end{aligned}
$$

where the notation $V_{\text {st }}=V\left(\left|\boldsymbol{r}_{t}-\boldsymbol{r}_{s}\right|\right)$ has been introduced for simplicity. Hence our approximation of taking $\exp \langle U\rangle$ in place of $\langle\exp U\rangle$ corresponds to neglecting all corelations between interaction terms in all orders.

This approximation gives for $d=2$

$$
P\left(\boldsymbol{S}^{2}\right) \propto \int_{\Gamma} \mathrm{d} x \underset{\sin ^{2} x}{x^{2}} \exp \left[-t x^{2} / 3-2 v( \pm i x)\right]
$$

A change such as the gloublar-coil transition is usually characterized by the singular behavior of eq 36 as a function of $v$. Here the following two facts are noted. In the case of $v=0$, the behavior of $\sin ^{2} x$ in the dominator of eq 36 as

$$
\sin ^{2} x \simeq \exp 2 R \quad(R \rightarrow \infty)
$$

along the integration path $\Gamma$ ensures the convergence of the $x$-integration.

In the case of $v \neq 0$, the interaction term $\exp [-2 v( \pm i x)]$ also behaves in such a manner as

$$
\exp [-2 v( \pm i x)] \simeq \exp -2 v R \quad(R \rightarrow \infty)
$$

Here, $R$ is the distance from the origin to a point on the path $\Gamma$ as shown in Figure 1.

Therefore the important mathematical facts which can be easily deduced from eq 37 and 38 are summarized as follows. If $v \geq 0$ (excluded volume), the integration with respect to $x$ along the path $\Gamma$ converges. For $0>v>-1$, the integral also converges, and the peak of $P(t)$ as a function of $v$ is pulled in as shown in Figure 2-4. At the same time, according to eq 18, i.e.,

$$
\alpha_{\mathrm{s}}^{2}=\frac{\left\langle\boldsymbol{S}^{2}\right\rangle}{\left\langle\boldsymbol{S}^{2}\right\rangle_{0}} \cong 1+2 v+v^{2}
$$

$\left\langle\boldsymbol{S}^{2}\right\rangle$ decreases smoothly with decreasing $v$ and become zero just where $v=-1$. In addition, the following quantity, whose logarithm is proportional to the free energy of a chain,

$$
\int P\left(S^{2}\right) \mathrm{d} S^{2} \propto(1+v)^{-2}
$$

diverges as $v \rightarrow-1$, where we have used the term $n=0$ only in eq 15 . These sketches appear in Figure 6. However, for $v \leq-1$, the $x$-integral does not converge, as is easily verified from eq 37 and 38 . These facts show qualitatively that the free energy diverges and that $\left\langle\boldsymbol{S}^{2}\right\rangle$ tends to approach zero as $v \rightarrow-1$, where the $x$-integral cannot converge; i.e., both the free energy and $\left\langle\boldsymbol{S}^{2}\right\rangle$ are analytic functions of $v$ for $\left.v\right\rangle-1$, but they are nonanalytic functions of $v$ at $v=-1$.

Other authors have put forth the theoretical prediction $^{5,6}$ that the mean square end-to-end 
Distribution Function of the Square Radius of Gyration

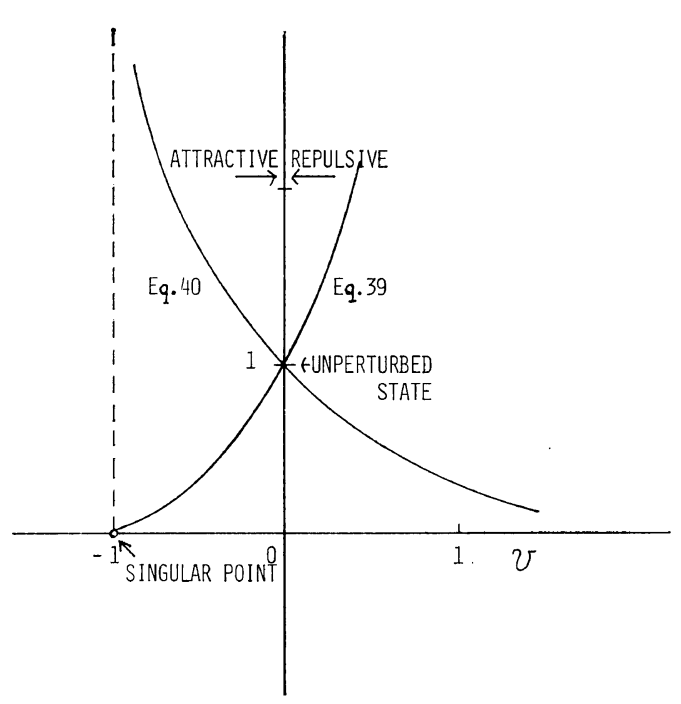

Figure 6. The behaviors of eq 39 and 40 as a function of $v$.

distance $\left\langle\boldsymbol{R}^{2}\right\rangle$ will decrease abruptly just below $v=0$. The present result for $\left\langle\boldsymbol{S}^{2}\right\rangle$ differs from this. However, since eq 39 is not an exact expression for $\left\langle\boldsymbol{S}^{2}\right\rangle$ within this present approximate treatment, it should not be concluded that $\left\langle\boldsymbol{S}^{2}\right\rangle$ tends toward zero as $v \rightarrow-1$. This is because Fujita and Norisue's method ${ }^{12}$ used for the derivation of eq 15 and 39 is not directly applicable where $v \simeq-1$; the discrepancy between the asymptotic form for $P(t)$, given by the leading term in eq 15, and the exact form, increases as $v \rightarrow-1$, (this discrepancy is about $8 \%$ for $v=0$, as shown in Appendix). Hence we cannot rule out completely the possibility that $\left\langle\boldsymbol{S}^{2}\right\rangle$ is positive finite at $v=-1$. In such a case there may occur a phase change in the polymer chain. It is a very difficult problem, to examine the behavior of the distribution function and $\left\langle S^{2}\right\rangle$ for the values of $v \simeq-1$, and this remains in the future problem.

Anyway, if some sort of singular behavior does occur, it will do so at the value, $v=-1$ according to our theory, and the critical value for the amplitude of the delta function will then be of the order of $l^{3} / L$. According to eq 19, $v$ being equal to 1 corresponds to $z=-2$. This value is much lower than that of Stockmayer's, i.e., $z^{*}=-0.14$ for $d=3$.

\section{SUMMARY}

In this paper we have obtained the twodimensional perturbed distribution functions of the square radius of gyration for a ring chain valid for small and large values of $t$, expressed by eq 15 and 16 , respectively. Using a simple variational technique, we have derived the expression for $\left\langle\boldsymbol{S}^{2}\right\rangle$ in a closed form. However, the minimization of the free energy at the stage of eq 31 requires further very careful examinations.

Moreover, for the chain with a purely attractive interaction we have shown that,

(1) there exists a negative critical value for the amplitude of the delta function potential; in other words, the excluded volume parameter $z$, below which the Fourier transformation of the characteristic function of the distribution of $S^{2}$ cannot be permitted.

(2) the free energy of the chain diverges and $\left\langle\boldsymbol{S}^{2}\right\rangle$ tends to zero as $z$ approaches the negative critical value of $z$ from above. However for this latter point, a rigorous proof cannot be given in this paper.

Finally, the present method cannot give information for the behavior of a three-dimensional chain in which we are more interested. As long as we are concerned with $P\left(\boldsymbol{S}^{2}\right)$ and $\left\langle\boldsymbol{S}^{2}\right\rangle$, it seems more appropriate ${ }^{8}$ to consider a twodimensional chain than a three-dimensional one.

Acknowledgments. The author would like to express his sincere appreciation to Dr. A. Hatano for his continual interest and helpful comments. My gratitude is due also to Dr. Y. Okabe for his kind suggestions in regard to the numerical calculations. The numerical calculations were made on the HITAC $8800 / 8700$ at Tokyo University.

\section{APPENDIX}

If we put $y=3(1+v)^{2} / t$, then eq 17 may be written as

$$
\begin{gathered}
\langle t\rangle=3(1+v)^{2} \int_{3 / 2}^{\infty} \mathrm{d} y\left(y^{1 / 2}-y^{-1 / 2}\right) \mathrm{e}^{-y} \\
/ \int_{3 / 2}^{\infty} \mathrm{d} y\left(y^{3 / 2}-y^{1 / 2}\right) \mathrm{e}^{-y}
\end{gathered}
$$

The two integrals in eq A1 are evaluated numerically, to give: 


$$
\langle t\rangle=0.919(1+v)^{2}
$$

The numerical factor 0.919 is due to our using the asymptotic expression for $P\left(S^{2}\right)$, which is valid for small values of $t$ in the calculation of $\langle t\rangle$. If the exact form for $P\left(S^{2}\right)$ is used, the value of 1 is obtained instead of 0.919 . This result means that the unperturbed mean square radius of gyration $\left\langle\boldsymbol{S}^{2}\right\rangle_{0}$ decreases effectively by ca. $8 \%$ through use of the leading term in eq 15 .

\section{REFERENCES}

1. T. Minato and A. Hatano, Polym. J., 9, 239 (1977) and references therein. In this paper numerical calculations were carried out only for a two-dimensional case, but they are also possible for one- and three-dimensional cases.

2. W. H. Stockmayer, Macromol. Chem., 35, 54 (1960).

3. Y. Chikahisa, J. Chem. Phys., 52, 206 (1970).

4. O. B. Ptitsyn, A. K. Kron and Yu. Ya. Eizner,
J. Polym. Sci., C16, 3509 (1968).

5. Y. Oono, J. Phy. Soc. Jpn., 39, 25 (1975).

6. N. Saito, Repts. Progr. Polym. Phys. Jpn., 17, 17 (1974).

7. T. Minato, submitted for publication in $J$. Phys. Soc. Jpn.

8. T. Minato and A. Hatano, J. Phys. Soc. Jpn., 42, 1992 (1977).

$K$. Šolc discussed the same problem from somewhat different point of view and obtained the asymptotic expressions of $\boldsymbol{P}\left(\boldsymbol{S}^{2}\right)$ valid for large values of $\boldsymbol{S}^{2}$, see,

K. Šolc, Macromolecules, 5, 705 (1972).

9. (a) M. Kurata and W. H. Stockmayer, Hortschr. Hochpolym. Forsch., 3, 196 (1963), (b) H. Yamakawa, "Modern Theory of Polymer Solutions," Harper and Row, New York, N.Y., 1971.

10. J. des Cloizeaux, J. Phys. (Paris), 31, 715 (1970).

11. C. Domb, J. Chem. Phys., 38, 2957 (1963); Adv. Chem. Phys., 15, 229 (1969).

12. H. Fujita and T. Norisue, J. Chem. Phys., 52, 1115 (1970). 